Mathematische Zeitschrift manuscript No.

(will be inserted by the editor)

\title{
Tight closure and linkage classes in Gorenstein rings
}

\author{
Adela Vraciu \\ Department of Mathematics, University of Kansas, Lawrence, KS 66045 \\ Received: date / Revised version: date
}

Abstract We study the relationship between the tight closure of an ideal and the sum of all ideals in its linkage class

\section{Introduction}

Tight closure and linkage have been developed as independent branches of commutative algebra. Tight closure was introduced by Hochster and Huneke in [HH1] as a tool for studying ideals in rings of positive characteristic, while the theory of linkage (liaison) has its roots in the study of curves in three dimensional projective space ( $\mathrm{Ap}], \mathrm{Ga}],[\mathrm{R}$, etc.). The algebraic foundations of linkage were established in PS2, [AN], [HU], et cetera.

The main result of this paper (Thm. 2) establishes a connection between these two theories. In the process of proving Thm. 2 we establish a relationship between ideals $J$ in the linkage class of an ideal $I$ and ideals in the linkage class of an $\mathbf{m}$-primary ideal $\left(I, x_{1}, \ldots, x_{d-g}\right)$, where $d=\operatorname{dim}(R), g=\operatorname{ht}(I)$, and the choice of $x_{1}, \ldots, x_{d-g}$ depends on $J$ (Prop. 6). This result might be of independent interest. We also develop a theory of corner powers of unmixed ideals, which are obtained as direct links of Frobenius powers. We explore some of their properties in Section 3, and we use them as a tool in the proof of Thm. 2.

\footnotetext{
* I thank Mel Hochster and Craig Huneke for their support and encouragement and for many helpful discussions.
} 
The setting is that of a Gorenstein ring of positive characteristic, where one can use the information available about the tight closure of ideals of finite projective dimension to relate the tight closure of an unmixed ideal to ideals in its linkage class.

The investigation carried out in this paper is motivated by the following question: If $R$ is a characteristic $p$ ring with test ideal $\tau$, and $I$ is an arbitrary ideal, what is the relationship between $I: \tau$ and $I^{*}$ ? The definition of the test ideal (see 2) implies that $I: \tau \supset I^{*}$, and in the particular case of a Gorenstein characteristic $p$ ring $R$ and an ideal $I$ of finite projective dimension we actually have equality: $I^{*}=I: \tau$ (see 1).

This equality is far from true in general. We propose to seek ideals that multiply $I: \tau$ into $I^{*}$. Note that in the case when $I: \tau$ is multiplied into $I^{*}$ by an $\mathbf{m}$-primary ideal, it follows that $I$ admits a test exponent, and therefore the tight closure of $I$ commutes with localization (see [HH2] for details about test exponents and localization of tight closure).

The main result of this paper (Thm. 2) states the following: if $R$ Gorenstein and $I$ is unmixed, then $(\tilde{I})^{*}(I: \tau) \subset I^{*}$, where $\tilde{I}$ denotes the sum of all the ideals in the linkage class of $I$. As an application, it follows that tight closure commutes with localization for any ideal $I$ for which $\tilde{I}$ is m-primary (Cor. 炄).

We do not expect that $\tilde{I}$ is the largest ideal with this property, but this result is interesting in light of the unexpected relationship between tight closure and linkage. There are several interesting consequences pertaining to properties of the linkage class of certain ideals. For instance, if $I$ is an unmixed tightly closed ideal containing the test ideal, then $I$ is maximal in its linkage class, in the sense of containing every ideal in its linkage class (see Cor. 2). This provides a class of examples addressing the question raised in [ [PU]: for which ideals $I$ is every ideal in the linkage class of $I$ contained in $I$ ?

\section{Preliminaries}

In this paper, $(R, m)$ denotes a Gorenstein local ring of characteristic $p>0$ and $q=p^{e}$ denotes a power of $p$. By parameter ideal we mean an ideal generated by part of a system of parameters.

We recall the relevant definitions:

Definition 1 Let $I$ be an ideal of $R$. For every $q=p^{e}, I^{[q]}:=\left(i^{q} \mid i \in\right.$ $I)$ is called the Frobenius qth power of $I . R^{0}$ denotes the set of elements in $R$ which are not in any minimal prime. 
An element $x \in R$ is in the tight closure of $I$ if there exists $c \in R^{0}$ such that $c x^{q} \in I^{[q]}$ for all $q=p^{e}$. We write $x \in I^{*}$.

Definition 2 The test ideal of $R$ is the ideal $\tau$ generated by all elements $c \in R^{0}$ such that for every ideal $I$ and every $x \in I^{*}$ we have $c x \in I$.

Definition 3 Let $(R, \mathbf{m})$ be a Noetherian local ring. An ideal I of height $g$ is called unmixed if all the associated prime ideals of I have height $g$.

Definition 4 An ideal $\mathbf{a}$ is called Gorenstein if the ring $R / \mathbf{a}$ is Gorenstein.

Note that if $R$ is a Gorenstein ring, then any parameter ideal is a Gorenstein ideal. If $R$ is Gorenstein and $R / \mathbf{a}$ has finite projective dimension over $R, R / \mathbf{a}$ is Gorenstein if and only if its minimal free resolution over $R$ is self-dual.

The following property of unmixed ideals is well-known:

Note 1 Let $(R, \mathbf{m})$ be a Noetherian local ring, let $I$ be an unmixed ideal of height $g$, and let $\mathbf{a} \subset I$ be a Gorenstein ideal of height $g$. Then $\mathbf{a}:(\mathbf{a}: I)=I$. If the assumption that $I$ is unmixed is removed, we have $\mathbf{a}:(\mathbf{a}: I)=I^{\text {unm }}$, where $I^{\text {unm }}$ denotes the intersection of the primary components in a primary decomposition of $I$ associated to primes of height $g$.

In this paper, linkage will mean linkage by Gorenstein ideals of finite projective dimension:

Definition 5 Let $I$ and $J$ be unmixed ideals of height $g$.

We say that $I$ and $J$ are directly linked if there is a Gorenstein ideal of finite projective dimension $\mathbf{a} \subset I \cap J$ of the same height $g$ such that $I=\mathbf{a}: J$ (and $J=\mathbf{a}: I$ ).

We say that $I$ and $J$ are linked (in $n$ steps), or that $J$ is in the linkage class of $I$, if there is a sequence of ideals $I_{1}=I, I_{2}, \ldots, I_{n+1}=$ $J$ such that $I_{i}$ and $I_{i+1}$ are directly linked for all $i=1, \ldots, n$. We denote the sum of all ideals in the linkage class of $I$ by $\tilde{I}$.

\section{Corner powers}

The corner powers of an unmixed ideal $I$, denoted $I^{<q>}$, are a tool used to study the relationship between the tight closure of an ideal and ideals in its linkage class; they are obtained as links of Frobenius powers. In this section we define the corner powers of unmixed ideals and study some of their properties. 
Definition 6 Let $(R, \mathbf{m})$ be a characteristic $p$ Gorenstein ring, and let $q$ be a power of $p$. If $I \subset R$ is an unmixed ideal of height $g$, let $\mathbf{a} \subset I$ be a Gorenstein ideal of finite projective dimension and of height $g$ (for example a parameter ideal) and let $J:=\mathbf{a}: I$. We define the qth corner power of $I$ to be the ideal $I^{<q>}:=\mathbf{a}^{[q]}: J^{[q]}$.

Note 2 Let $R, I, \mathbf{a}, J$ be as above. If moreover $I$ is $\mathbf{m}$-primary, we can also write $J^{[q]}=\mathbf{a}^{[q]}: I^{<q>}$ by Note. 1, since $J^{[q]}$ is unmixed and $\mathbf{a}^{[q]}$ is Gorenstein. However, this equality is not true in general for non m-primary ideals, because even though $J$ is unmixed, $J^{[q]}$ might not be unmixed. We can say in general that $\mathbf{a}^{[q]}: I^{<q>}=\left(J^{[q]}\right)^{\text {unm }}$.

Also note that if $I$ is $\mathbf{m}$-primary, we have $I^{<p q>}=\left(I^{<q>}\right)^{<p>}$, since $J^{[q]}=\mathbf{a}^{[q]}: I^{<q>} \Rightarrow\left(I^{<q>}\right)^{<p>}=\left(\mathbf{a}^{[q]}\right)^{[p]}:\left(J^{[q]}\right)^{[p]}=\mathbf{a}^{[p q]}$ : $J^{[p q]}=I^{<p q>}$. We do not know if this equality is true for unmixed ideals which are not $\mathbf{m}$-primary.

Note that a priori the definition of $I^{<q>}$ depends on the choice of a. In order to show that $I^{<q>}$ is well-defined we need the following preliminary results:

Lemma 1 Let $R$ be a Gorenstein local ring and let $\mathbf{a} \subset \mathbf{b}$ be Gorenstein ideals of the same height $g$ and of finite projective dimension.

The natural map $R / \mathbf{a} \longrightarrow R / \mathbf{b}$ extends to a map of complexes $\psi$. from a minimal free resolution of $R / \mathbf{a}$ to a minimal free resolution of $R / \mathbf{b}$. Let $\delta \in R$ be such that the last map $\psi_{g}: R \longrightarrow R$ is multiplication by $\delta$.

Then $\mathbf{a}: \mathbf{b}=(\mathbf{a}, \delta)$, and $\mathbf{b}=\mathbf{a}: \delta$.

Proof Prop.2.6 in [PS2] shows that the mapping cone of the map $\psi$. is the projective free resolution of $R /(\mathbf{a}: \mathbf{b})$. Since the first map in the mapping cone complex is given by $\left(a_{1}, \ldots, a_{g}, \delta\right)$, where $\mathbf{a}=$ $\left(a_{1}, \ldots, a_{g}\right)$, it follows that $\mathbf{a}: \mathbf{b}=(\mathbf{a}, \delta)$, and therefore $\mathbf{a}: \delta=\mathbf{b}$ by Note. 1.

Lemma 2 Let $(R, \mathbf{m})$ be a Gorenstein ring, I an unmixed ideal of height $g$, and let $\mathbf{a} \subset \mathbf{b} \subset I$ be Gorenstein ideals of height $g$ and finite projective dimension. Let $\delta$ be as in Lemma 1 . Then the ideal $(\mathbf{a}, \delta I)$ is unmixed.

Proof We have the following short exact sequence:

$$
0 \longrightarrow \frac{(\mathbf{a}, \delta)}{(\mathbf{a}, \delta I)} \longrightarrow \frac{R}{(\mathbf{a}, \delta I)} \longrightarrow \frac{R}{(\mathbf{a}, \delta)} \longrightarrow 0 .
$$

The first term in the short exact sequence is isomorphic to

$$
\frac{R}{(\mathbf{a}, \delta I): \delta}
$$


If $u \in(\mathbf{a}: \delta I): \delta$, we can write $\delta(u-i) \in \mathbf{a}$ for some $i \in I$, hence $u-i \in \mathbf{a}: \delta=\mathbf{b} \subset I$. This shows that the first term in the short exact sequence is isomorphic to $R / I$, hence unmixed, while the last term is $R /(\mathbf{a}: \mathbf{b})$, which is also unmixed. Therefore the middle term, $R /(\mathbf{a}+\delta I)$ is unmixed, which finishes the proof of the lemma.

Proposition 1 Let $(R, \mathbf{m})$ be a Gorenstein ring of characteristic $p$. The corner powers of an unmixed ideal I are well-defined.

Proof Let $\mathbf{a}, \mathbf{b} \subset I$ be Gorenstein ideal of finite projective dimensions of height $g$. Without loss of generality, we can assume $\mathbf{a} \subset \mathbf{b}$, since otherwise we may replace $\mathbf{a}$ by an $\mathbf{a}^{\prime} \subset \mathbf{a} \cap \mathbf{b}$. Let $\delta$ be as in Lemma 1, and let

$$
J_{1}=\mathbf{a}: I, \quad J_{2}=\mathbf{b}: I=(\mathbf{a}: \delta): I=\mathbf{a}: \delta I .
$$

Note that we can also write

$$
I=\mathbf{b}: J_{2}=\mathbf{a}: \delta J_{2}
$$

Note that $\mathbf{b}^{[q]}$ (respectively $\mathbf{a}^{[q]}$ ) is again a Gorenstein ideal, and a free resolution of $R / \mathbf{b}^{q}$ (respectively $R / \mathbf{a}^{[q]}$ ) is obtained from a free resolution of $R / \mathbf{b}$ (respectively $R / \mathbf{a}$ ) by raising all the entries of the matrices appearing in the free resolution to the $q$ th power (by Thm. 1.7 in [PS1]). Lemma 1 applied to $\mathbf{a}^{[q]} \subset \mathbf{b}^{[q]}$ shows that $\mathbf{b}^{[q]}=\mathbf{a}^{[q]}: \delta^{q}$.

We need to show that for all $q=p^{e}$, we have $\mathbf{a}^{[q]}: J_{1}^{[q]}=\mathbf{b}^{[q]}: J_{2}^{[q]}$. Note that $\mathbf{b}^{[q]}: J_{2}^{[q]}=\mathbf{a}^{[q]}: \delta^{q} J_{2}^{[q]}$, and therefore it suffices to show that $\left(\mathbf{a}^{[q]}, \delta^{q} J_{2}^{[q]}\right)=\left(\mathbf{a}^{[q]}, J_{1}^{[q]}\right)$. This is obviously true, since equation 2 combined with Note. 1 shows $\left(\mathbf{a}, \delta J_{2}\right)=\left(\mathbf{a}, J_{1}\right)=\mathbf{a}: I$ (note that $\left(\mathbf{a}, J_{1}\right)=J_{1}$ and $J_{2}$ are unmixed by construction, and $\left(\mathbf{a}, \delta J_{2}\right)$ is unmixed by Lemma 2).

We would like to see how the corner powers $I^{<q>}$ are related to the Frobenius powers $I^{[q]}$ :

Proposition $\mathbf{2}$ Let $(R, \mathbf{m})$ be a Gorenstein characteristic $p$ ring and let $I$ be an unmixed ideal. For all $q=p^{e}$ we have $I^{[q]} \subset I^{<q>}$ and if $I$ has finite projective dimension we have equality. If $R$ is a hypersurface the equality holds for all $q$ if and only if I has finite projective dimension.

Proof Choose $\mathbf{a} \subset I$ a parameter ideal and let $J=\mathbf{a}: I=\left(f_{1}, \ldots, f_{n}\right)$. To show that $I^{[q]} \subset I^{<q>}$ note that $I^{[q]}=(\mathbf{a}: J)^{[q]} \subset \mathbf{a}^{[q]}: J^{[q]}$, because $I^{[q]} J^{[q]}=(\mathbf{a}: J)^{[q]} J^{[q]}=((\mathbf{a}: J) J)^{[q]} \subset \mathbf{a}^{[q]}$. 
Consider the short exact sequence

$$
0 \longrightarrow \frac{R}{I} \stackrel{\left(f_{1}, \ldots, f_{n}\right)}{\longrightarrow} \bigoplus_{n} \frac{R}{\mathbf{a}} \longrightarrow N \longrightarrow 0
$$

where $N$ is the cokernel of the map given by $\left(f_{1}, \ldots, f_{n}\right)$. Recall that $R^{1 / q}$ denotes the $R$-algebra obtained by adjoining all $q$ th roots of elements in $R$, and it can be identified with $R$ via the action of the Frobenius map $x \rightarrow x^{q}$. Tensoring this short exact sequence with $R^{1 / q}$ yields

$0 \longrightarrow \operatorname{Tor}_{1}^{R}\left(N, R^{1 / q}\right) \longrightarrow \frac{R^{1 / q}}{I R^{1 / q}} \stackrel{\left(f_{1}, \ldots, f_{n}\right)}{\longrightarrow} \bigoplus_{n} \frac{R^{1 / q}}{\mathbf{a} R^{1 / q}} \longrightarrow N \otimes R^{1 / q} \longrightarrow 0$

and therefore $\operatorname{Tor}_{1}^{R}\left(N, R^{1 / q}\right)$ is isomorphic to

$$
\frac{\mathbf{a} R^{1 / q}:_{R^{1 / q}}\left(f_{1}, \ldots, f_{n}\right)}{I R^{1 / q}},
$$

which, upon identifying $R^{1 / q}$ with $R$, can be identified with

$$
\frac{\mathbf{a}^{[q]}:\left(f_{1}^{q}, \ldots, f_{n}^{q}\right)}{I^{[q]}}=\frac{I^{<q>}}{I^{[q]}} .
$$

If $I$ has finite projective dimension, it follows that $N$ also has finite projective dimension, and therefore $\operatorname{Tor}_{1}^{R}\left(N, R^{1 / q}\right)=0$ (see Thm. 1.7 in [PS1]), and the short exact sequence shows that $I^{[q]}=I^{<q>}$.

If $R$ is a hypersurface and $I^{[q]}=I^{<q>}$ for all $q$, it follows that $\operatorname{Tor}_{1}^{R}\left(N, R^{1 / q}\right)=0$ for all $q$, which implies that $N$ has finite projective dimension by $[\mathrm{He}$, and therefore $I$ has finite projective dimension.

The following property of corner powers is essential for the purpose of this paper:

Theorem 1 Let $(R, \mathbf{m})$ be a Gorenstein ring of characteristic $p$, with test ideal $\tau$.

a). If $\mathbf{a}$ is an ideal of finite projective dimension, then $\mathbf{a}^{*}=\mathbf{a}: \tau$.

b). If I is an unmixed ideal, then for all $q=p^{e}$ we have

$$
I^{<q>}: \tau \supset(I: \tau)^{[q]} .
$$

More precisely:

$$
\begin{aligned}
I: \tau & =\left\{x \in R \mid c x^{q} \in I^{<q>} \text { for some } c \in R^{0} \text { and all } q=p^{e}\right\} \\
& =\left\{x \in R \mid c x^{q} \in I^{<q>} \text { for all } c \in \tau \text { and all } q=p^{e}\right\}
\end{aligned}
$$


Proof The statement in part a). for the case when $\mathbf{a}$ is a parameter ideal is Cor.4.2(2) in [Hu1]. The general case will follow from part b).

To prove part b)., choose a $\subset I$ a parameter ideal of the same height as $I$ and let $c \in \tau$. We have: $c x^{q} \in I^{<q>} \Longleftrightarrow c x^{q} \in \mathbf{a}^{[q]}$ : $J^{[q]} \Longleftrightarrow c x^{q} J^{[q]} \subset \mathbf{a}^{[q]}$. This holds for all $q$ if and only if $x J \subset \mathbf{a}^{*}=$ $\mathbf{a}: \tau \Longleftrightarrow x \in \mathbf{a}: \tau J=I: \tau$.

For the general case of part a)., use the fact that $\mathbf{a}^{[q]}=\mathbf{a}^{<q>}$ for any $\mathbf{a}$ of finite projective dimension (see 2), and therefore for any $u \in \mathbf{a}: \tau$ we have $\tau u^{q} \subset \mathbf{a}^{<q>}=\mathbf{a}^{[q]}$, hence $u \in \mathbf{a}^{*}$.

Proposition 3 Let $(R, m)$ be a Gorenstein local ring. If $J$ is an $m$ primary ideal, then $l\left(R / J^{<q>}\right)$ behaves like a Hilbert-Kunz function, that is, there is a real constant $c_{J}>0$ such that

$$
l\left(\frac{R}{J<q>}\right)=c_{J} q^{d}+\mathcal{O}\left(q^{d-1}\right),
$$

where $d=\operatorname{dim} R$. More precisely, we have

$$
l\left(R / J^{<q>}\right)=l\left(R / \mathbf{a}^{[q]}\right)-l\left(R / I^{[q]}\right),
$$

where $\mathbf{a} \subset J$ is a Gorenstein ideal of finite projective dimension, and $I=\mathbf{a}: J$.

Proof We have $J^{<q>}=\mathbf{a}^{[q]}: I^{[q]}$. Note that $R / \mathbf{a}^{[q]}$ maps onto $R / I^{[q]}$.

Since $R$ is Gorenstein and $\mathbf{a}$ is a Gorenstein ideal of finite projective dimension, $R / \mathbf{a}^{[q]}$ is a Gorenstein ring; hence we have

$$
\frac{R}{\mathbf{a}^{[q]}}=E_{R / \mathbf{a}^{[q]}}(k) \Rightarrow \frac{J^{<q>}}{\mathbf{a}^{[q]}}=\operatorname{Ann}_{R / \mathbf{a}^{[q]}}\left(\frac{I^{[q]}}{\mathbf{a}^{[q]}}\right)=E_{R / I^{[q]}}(k),
$$

and therefore

$$
l\left(\frac{J^{<q>}}{\mathbf{a}^{[q]}}\right)=l\left(\frac{R}{I^{[q]}}\right) .
$$

This can be rewritten as

$$
l\left(\frac{R}{\mathbf{a}^{[q]}}\right)-l\left(\frac{R}{J^{<q>}}\right)=l\left(\frac{R}{I^{[q]}}\right),
$$

and therefore $l\left(R / J^{<q>}\right)$ can be written as a difference of HilbertKunz functions (see [Md]).

Proposition 4 Let $(R, m)$ be a Gorenstein local ring with test ideal $\tau$. Assume that $R$ is excellent and analytically irreducible.

a). If $I \supset \tau$, then for all $q, I^{<q>} \supset \tau$.

b). If $I$ is an ideal strictly contained in $\tau$, then there is a $q_{0}$ such that $I^{<q>} \subset m^{\left[q / q_{0}\right]}$ for all $q \geq q_{0}$. 
Proof a). Let $\mathbf{a} \subset I$ be a Gorenstein ideal of finite projective dimension and let $J=\mathbf{a}: I \subset \mathbf{a}: \tau$. Using the fact that $\mathbf{a}^{*}=\mathbf{a}: \tau$, and therefore $(\mathbf{a}: \tau)^{[q]} \subset \mathbf{a}^{[q]}: \tau$, we have

$$
I^{<q>}=\mathbf{a}^{[q]}: J^{[q]} \supset \mathbf{a}^{[q]}:(\mathbf{a}: \tau)^{[q]} \supset \mathbf{a}^{[q]}:\left(\mathbf{a}^{[q]}: \tau\right) \supset \tau .
$$

b). Let $\mathbf{a} \subset I$ be a Gorenstein ideal of finite projective dimension, and let $J=\mathbf{a}: I$. The assumptions imply that $\mathbf{a}: \tau=\mathbf{a}^{*}$ is strictly contained in $J$. Let $f \in J \backslash \mathbf{a}^{*}$. Then the Prop. 2.4 in Ab shows that there is a $q_{0}$ such that

$$
I^{<q>}=\mathbf{a}^{[q]}: J^{[q]} \subset \mathbf{a}^{[q]}: f^{q} \subset m^{\left[q / q_{0}\right]}
$$

Example 1 It is not always true that $I^{<q>} \subset I$. Let

$$
R=k[[X, Y, Z]] /\left(X^{3}+Y^{3}+Z^{3}\right), p=\operatorname{char}(k)=2, I=\left(x^{2}, y^{2}, z^{2}\right)
$$
and $\mathbf{a}=\left(x^{2}, y^{2}\right)$. Then

$$
J=\left(x^{2}, y^{2}\right): I=\left(x^{2}, y^{2}\right): z^{2}=\left(x^{2}, y^{2}, z\right) .
$$

Then $I^{<2>}=\left(x^{4}, y^{4}\right): z^{2}=\left(x^{4}, y^{4}, x y z\right)$, and we have $x y z \in I^{<2>} \backslash I$.

The usefulness of corner powers is further illustrated in the following proposition (compare to exercise 2.8 in [Hu2] and Prop. 3.3(d) in [HH2]):

Proposition 5 Let $R$ be a Gorenstein ring of characteristic p with test ideal $\tau$ and let $I$ be an arbitrary ideal. For all $q=p^{e}$, let $I_{q}:=$ $\left\{u \in R \mid u^{q} \subset \tau I^{[q]}: \tau\right\}$. Then for all $q$ we have $I_{p q} \subset I_{q}$, and therefore $I^{*}$ can be written as a nonincreasing intersection of the ideals $I_{q}$.

Proof First assume that $I$ is $\mathbf{m}$-primary, so that if $\mathbf{a} \subset I$ is an $\mathbf{m}$ primary parameter ideal and $J=\mathbf{a}: I$ we have $I^{[q]}=\mathbf{a}^{[q]}: J^{<q>}$.

Let $u \in I_{p q}$, so $\tau u^{p q} \in \tau I^{[p q]}$. Since $J^{<p q>}=\mathbf{a}^{[p q]}: I^{[p q]}$, and $J^{<p q>}: \tau=\mathbf{a}^{[p q]}: \tau I^{[p q]}$, this implies that $\tau u^{p q}\left(J^{<p q>}: \tau\right) \subset \mathbf{a}^{[p q]}$. Using Thm. 1(b) applied to $J^{<q>}$, we get

$$
u^{p q}\left(J^{<q>}: \tau\right)^{[p]} \subset u^{p q}\left(J^{<p q>}: \tau\right) \subset \mathbf{a}^{p q}: \tau=\left(\mathbf{a}^{[p q]}\right)^{*},
$$

which implies that

$$
u^{q}\left(J^{<q>}: \tau\right) \subset\left(\mathbf{a}^{[q]}\right)^{*}=\mathbf{a}^{[q]}: \tau,
$$

and therefore $\tau u^{q} \in \mathbf{a}^{[q]}:\left(J^{<q>}: \tau\right)=\mathbf{a}^{[q]}+\tau I^{[q]}$ (the last equality follows because $\mathbf{a}^{[q]}:\left(\mathbf{a}^{[q]}+\tau I^{[q]}\right)=\left(\mathbf{a}^{[q]}: I^{[q]}\right): \tau=J^{<q>}: \tau$, and $\mathbf{a}^{[q]}+\tau I^{[q]}$ is an unmixed ideal - being $\mathbf{m}$-primary - so we can use Note. 1). Since this holds for any parameter ideal $\mathbf{a} \subset I$, we get $\tau u^{q} \in \tau I^{[q]}$ by Krull's intersection theorem. 
If $I$ is not $\mathbf{m}$-primary and $u^{p q} \in \tau I^{[p q]}: \tau$, we have $u^{p q} \in \tau(I+$ $\left.\mathbf{m}^{t}\right)^{[p q]}: \tau$ for all $t>0$, which according to the $\mathbf{m}$-primary case implies

$u^{q} \in \tau\left(I+\mathbf{m}^{t}\right)^{[q]}: \tau$ for all $t$, hence intersecting over all $t>0$ yields $u^{q} \in \tau I^{[q]}: \tau$.

In order to justify the last sentence of the proposition, note that $\cap_{q} I_{q} \subset I^{*}$ by the definition of tight closure, and vice versa $I^{*} \subset I_{q}$ for all $q$, since $u \in I^{*} \Rightarrow u^{q} \in\left(I^{[q]}\right)^{*} \subset \tau I^{[q]}: \tau$ (the last implication follows by Thm.3.1 in $[\mathrm{Vr})$.

Corollary 1 Let $(R, \mathbf{m})$ be a Gorenstein ring with test ideal $\tau$ and let $I$ be an ideal such that $(I: \tau) / I^{*}$ has finite length. Then $I$ admits a test exponent, i.e. there exists a $q_{0}=p^{e_{0}}$ such that if $\tau x^{q_{0}} \in \tau I^{\left[q_{0}\right]}$ with $x \in R$, then $x \in I^{*}$. In particular, tight closure commutes with localization for $I$.

Proof According to Prop. 5, the assumption that $(I: \tau) / I^{*}$ has finite length implies that $I^{*}=I_{q_{0}}$ for some $q_{0}$, since $I^{*}$ can be written as a nonincreasing intersection of the ideals $I_{q}$, which are contained in $I_{1}=\tau I: \tau \subset I: \tau$.

Note 3 The notion of test exponent used here is a modification of the notion introduced in [HH2], which basically states that $\tau x^{q_{0}} \in$ $I^{\left[q_{0}\right]} \Rightarrow x \in I^{*}$. The condition used here is weaker, but still sufficient to guarantee that tight closure commutes with localization in the context of Cor. 1, using the fact that in a Gorenstein local ring the test ideal commutes with localization (Thm.4.1 in [Sm] and it is a strong test ideal (Thm.3.1 in $[\mathrm{Vr}]$ ). The proof follows along the lines of Prop.2.3 in [HH2]: if $W \subset R$ is a multiplicative system and $u / 1 \in\left(I_{W}\right)^{*}$, then we have $\tau u^{q_{0}} / 1 \in \tau\left(I_{W}\right)^{\left[q_{0}\right]}$, and we can choose $f \in W$ such that $f \tau u^{q_{0}} \in \tau I^{\left[q_{0}\right]}$, so $\tau(f u)^{q_{0}} \in \tau I^{\left[q_{0}\right]}$. But then $f u \in I^{*}$, and so $u \in\left(I^{*}\right)_{W}$.

\section{Main result}

The following theorem is the main result of this paper:

Theorem 2 Let $(R, \mathbf{m})$ be a Gorenstein local ring with test ideal $\tau$. Let $I$ be an unmixed ideal, and let denote $\tilde{I}$ the sum of all ideals in the linkage class of $I$.

Then we have

$$
(\tilde{I})^{*}(I: \tau) \subset I^{*}
$$


The outline of the proof is as follows: we prove the result for the case when $I$ is $\mathbf{m}$-primary, then we reduce to the $\mathbf{m}$-primary case via adjoining extra elements to all the ideals involved. Our proof in the $\mathbf{m}$-primary case rests on the ability to exploit the relationships between corner powers and Frobenius powers of the ideal $I$, which in turn rests on the fact that for every $q, I^{[q]}$ is still an unmixed ideal (this is obvious if $I$ is $\mathbf{m}$-primary, but rarely true otherwise).

In order to complete the announced reduction to the $\mathbf{m}$-primary case, we establish a relationship between the linkage class of an ideal $I$ of height $g$ and the linkage class of an $\mathbf{m}$-primary ideal containing $I$ (see Prop. 6).

To this end we need the following preliminary result:

Lemma 3 Let $(R, \mathbf{m})$ be a Gorenstein ring, I an unmixed ideal of height $g$, and let $\mathbf{a} \subset \mathbf{b} \subset I$ be Gorenstein ideals of height $g$ and finite projective dimension.

Then we have

$$
\mathbf{a}:(\mathbf{b}: I)=\mathbf{a}+I(\mathbf{a}: \mathbf{b}) \text {. }
$$

Proof We need to check that the ideal on the right hand side is unmixed and that the equality holds after taking duals into a. Indeed,

$$
\mathbf{a}:[\mathbf{a}:(\mathbf{b}: I)]=\mathbf{b}: I,
$$

and

$$
\mathbf{a}:[\mathbf{a}+I(\mathbf{a}: \mathbf{b})]=[\mathbf{a}:(\mathbf{a}: \mathbf{b})]: I=\mathbf{b}: I,
$$

because $\mathbf{a} \subset \mathbf{b}$. The fact that $\mathbf{a}+I(\mathbf{a}: \mathbf{b})$ is unmixed follows from Lemma 2 .

Proposition 6 Let $(R, \mathbf{m})$ be a local Gorenstein ring and let $I$ be an unmixed ideal of height $g<d:=\operatorname{dim}(R)$. Then for every ideal $J$ in the linkage class of $I$ there exist elements $x_{1}, \ldots, x_{d-g}$ such that $\left(I, x_{1}, \ldots, x_{d-g}\right)$ is $\mathbf{m}$-primary, and for all $t>0$ there exists an ideal $J_{t}$ in the linkage class of $I_{t}:=\left(I, x_{1}^{t}, \ldots, x_{d-g}^{t}\right)$ with $J \subset J_{t}$.

Proof Let $J$ be an ideal in the linkage class of $I$,

$$
J=\mathbf{a}_{n}:\left(\mathbf{a}_{n-1}: \ldots:\left(\mathbf{a}_{1}: I\right)\right),
$$

with $\mathbf{a}_{1} \subset I$ and $\mathbf{a}_{i} \subset \mathbf{a}_{i-1}:\left(\mathbf{a}_{i-2}: \ldots:\left(\mathbf{a}_{1}: I\right)\right)$ for $1<i \leq n$, Gorenstein ideals of finite projective dimension and of height $g$. Choose $\mathbf{b} \subset \mathbf{a}_{1} \cap \ldots \cap \mathbf{a}_{n}$ a parameter ideal of height $g$, and choose $\underline{x}=$ $x_{1}, \ldots, x_{d-g}$ a sys tem of parameters modulo $\mathbf{b}$ (and hence also a 
system of parameters modulo each of the $\mathbf{a}_{i}$ 's). Then for all $t>0$, we claim that

$$
J_{t}:=\left(\mathbf{a}_{n}, \underline{x}^{t}\right):\left(\left(\mathbf{a}_{n-1}, \underline{x}^{t}\right): \ldots\left(\left(\mathbf{a}_{1}, \underline{x}^{t}\right):\left(I, \underline{x}^{t}\right)\right)\right)
$$

is in the linkage class of $\left(I, \underline{x}^{t}\right)$, and $J \subset J_{t}$. Here we use the notation $\underline{x}^{t}=x_{1}^{t}, \ldots, x_{d-g}^{t}$.

First observe that for every $i=1, \ldots, n$ and for every $t$, the ideal $\left(\mathbf{a}_{i}, \underline{x}^{t}\right)$ is still a Gorenstein ideal of finite projective dimension. This is justified by the fact that if $\mathbf{F}{ }_{.}$denotes a minimal free resolution of $R / \mathbf{a}_{i}$, and K. $\left(\underline{x}^{t}\right)$ denotes the Koszul complex of $\underline{x}^{t}$, then $\mathbf{F} \cdot{ }_{\cdot} \otimes \mathbf{K} .\left(\underline{x}^{t}\right)$ is a free resolution of $R /\left(\mathbf{a}_{i}, \underline{x}^{t}\right)$. Indeed, it is enough to see that $\mathbf{F}_{\cdot i} \otimes \mathbf{K} .\left(\underline{x}^{t}\right)$ is acyclic. This is true because its $i$ th homology module is $\operatorname{Tor}_{i}^{R}\left(R / \mathbf{a}_{i}, R /\left(\underline{x}^{t}\right)\right)$, which is zero for all $i \geq 1$ since $\mathbf{K}$. $\left(\underline{x}^{t}\right)$ remains acyclic upon tensoring with $R / \mathbf{a}_{i}$ (the $x$ 's form a regular sequence in $\left.R / \mathbf{a}_{i}\right)$.

We prove the claim by induction on $n$. The case $n=1$ is obvious. Let $n=2$.

According to Lemma 1, we can write $\mathbf{a}_{2}=\mathbf{b}: \delta$ where $\delta \in R$ is such that the last map $\psi_{g}: R \longrightarrow R$ in the map of complexes $\psi$. from the Koszul resolution of $R / \mathbf{b}$ to a minimal free resolution of $R / \mathbf{a}_{2}$ is multiplication by $\delta$. Since the corresponding free resolutions for $R /\left(\mathbf{b}, \underline{x}^{t}\right)$ and $R /\left(\mathbf{a}_{2}, \underline{x}^{t}\right)$ are obtained from the resolutions of $R / \mathbf{b}$, respectively $R / \mathbf{a}_{2}$, by tensoring with $\mathbf{K} .\left(\underline{x}^{t}\right)$, it follows that the last map of the map of complexes between them is again multiplication by $\delta$, and therefore we also have $\left(\mathbf{a}_{2}, \underline{x}^{t}\right)=\left(\mathbf{b}, \underline{x}^{t}\right): \delta$. It is enough to show that

$$
\left(\mathbf{b}, \underline{x}^{t}\right):\left(\left(\mathbf{a}_{1}, \underline{x}^{t}\right):\left(I, \underline{x}^{t}\right)\right) \supset \mathbf{b}:\left(\mathbf{a}_{1}: I\right),
$$

because then it follows that

$$
\begin{aligned}
& \left(\mathbf{a}_{2}, \underline{x}^{t}\right):\left[\left(\mathbf{a}_{1}, \underline{x}^{t}\right):\left(I, \underline{x}^{t}\right)\right]=\left[\left(\mathbf{b}, \underline{x}^{t}\right): \delta\right]:\left[\left(\mathbf{a}_{1}, \underline{x}^{t}\right):\left(I, \underline{x}^{t}\right)\right]= \\
& {\left[\left(\mathbf{b}, \underline{x}^{t}\right):\left(\left(\mathbf{a}_{1}, \underline{x}^{t}\right):\left(I, \underline{x}^{t}\right)\right)\right]: \delta \supset} \\
& {\left[\mathbf{b}:\left(\mathbf{a}_{1}: I\right)\right]: \delta=(\mathbf{b}: \delta):\left(\mathbf{a}_{1}: I\right)=\mathbf{a}_{2}:\left(\mathbf{a}_{1}: \delta\right) .}
\end{aligned}
$$

By replacing $\mathbf{a}_{2}$ by $\mathbf{b}$ and changing the notation accordingly, we can assume without loss of generality that $\mathbf{a}_{2} \subset \mathbf{a}_{1}$. By Lemma 3, in this case we have

$$
\begin{gathered}
\mathbf{a}_{2}:\left(\mathbf{a}_{1}: I\right)=\mathbf{a}_{2}+ \\
=\left(\mathbf{a}_{2}: \mathbf{a}_{1}\right) \subset\left(\mathbf{a}_{2}, \underline{x}^{t}\right)+\left(I, \underline{x}^{t}\right)\left(\left(\mathbf{a}_{2}, \underline{x}^{t}\right):\left(\mathbf{a}_{1}, \underline{x}^{t}\right)\right) \\
=\left(\mathbf{a}_{2}, \underline{x}^{t}\right):\left(\left(\mathbf{a}_{1}, \underline{x}^{t}\right):\left(I, \underline{x}^{t}\right)\right) .
\end{gathered}
$$


This finishes the proof of the claim for the case $n=2$. Assume $n \geq 3$. Let $K:=\mathbf{a}_{n-2}:\left(\ldots\left(\mathbf{a}_{1}: I\right)\right)$ and $K_{t}:=\left(\mathbf{a}_{n-2}, \underline{x}^{t}\right):\left(\ldots\left(\left(\mathbf{a}_{1}, \underline{x}^{t}\right):\left(I, \underline{x}^{t}\right)\right)\right)$.

The induction hypothesis shows that $K \subset K_{t}$ for all $t$, hence $\left(K, \underline{x}^{t}\right) \subset K_{t}$. Therefore we have

$$
J_{t}=\left(\mathbf{a}_{n}, \underline{x}^{t}\right):\left[\left(\mathbf{a}_{n-1}, \underline{x}^{t}\right): K_{t}\right] \supset\left(\mathbf{a}_{n}, \underline{x}^{t}\right):\left[\left(\mathbf{a}_{n-1}, \underline{x}^{t}\right):\left(K, \underline{x}^{t}\right)\right],
$$

since double linkage preserves inclusions, and by the case $n=2$ applied to $I:=K$ it follows that

$$
J_{t} \supset \mathbf{a}_{n}:\left(\mathbf{a}_{n-1}: K\right)=J .
$$

This finishes the proof of the claim and the proof of the proposition.

We are now ready to prove Thm. 2:

Proof It is enough to show that $\tilde{I}(I: \tau) \subset I^{*}$, since this will imply that the tight closure of the ideal on the left hand side is contained in $I^{*}$, hence $(\tilde{I})^{*}(I: \tau) \subset(\tilde{I}(I: \tau))^{*} \subset I^{*}$.

First assume that $I$ is $\mathbf{m}$-primary.

Theorem 11 implies that if $d \in R$ is such that $d^{q} I^{<q>} \subset I^{[q]}$ for all $q$, then $d(I: \tau) \subset I^{*}$. We claim that any $d \in \tilde{I}$ has this property.

It is obvious that any $d \in I$ will have the desired property. In order to finish the proof of the claim, it suffices to prove that for any $\mathbf{a} \subset I$ Gorenstein ideal of finite projective dimension, for all $c \in R$ and for all $q$ we have $c I^{<q>} \subset I^{[q]} \Longleftrightarrow c J^{<q>} \subset J^{[q]}$, where $J=\mathbf{a}: I$.

Indeed, we have

$$
\begin{gathered}
c I^{<q>} \subset I^{[q]} \Longleftrightarrow c\left(\mathbf{a}^{[q]}: J^{[q]}\right) \subset \mathbf{a}^{[q]}: J^{<q>} \Longleftrightarrow \\
\mathbf{a}^{[q]}: J^{[q]} \subset \mathbf{a}^{[q]}: c J^{<q>} \Longleftrightarrow c J^{<q>} \subset J^{[q]} .
\end{gathered}
$$

Here we used the fact that $I^{[q]}=\mathbf{a}^{[q]}: J^{<q>}$, since $I^{[q]}$ is $\mathbf{m}$-primary. This shows that for every $K$ in the linkage class of $I$ we have $c I^{<q>} \subset$ $I^{[q]} \Longleftrightarrow c K^{<q>} \subset K^{[q]}$, and therefore for every $d \in K$ we have $d^{q} I^{<q>} \subset I^{[q]}$.

Let $u \in \tilde{I}$ and write $u=d_{1}^{q}+\ldots d_{n}^{q}$ with $d_{i} \in K_{i}$, where $K_{1}, \ldots K_{n}$ are ideals in the linkage class of $I$. Since $d_{i}^{q} I^{<q>} \subset I^{[q]}$ for all $i$, we have $u^{q} I^{<q>} \subset I^{[q]}$. If $g \in I: \tau$, it follows that $\tau g^{q} \in I^{<q>}$, and hence $\tau u^{q} g^{q} \in I^{[q]}$, which shows that $u g \in I^{*}$ as desired.

Now assume that $I$ has height $g<d=\operatorname{dim}(R)$, and let $J \subset \tilde{I}$ be an ideal in the linkage class of $I$. Let $\underline{x}=x_{1}, \ldots, x_{d-g}$ and $J_{t}$ be as in Prop. 6, for all $t>0$. According to the m-primary case, we have:

$$
J\left(\left(I, \underline{x}^{t}\right): \tau\right) \subset J_{t}\left(\left(I, \underline{x}^{t}\right): \tau\right) \subset\left(I, \underline{x}^{t}\right)^{*}
$$


for all $t$. Intersect over all $t$ and use the fact that

$$
\bigcap_{t}\left(\left(I, \underline{x}^{t}\right): \tau\right)=\left(\bigcap_{t}\left(I, \underline{x}^{t}\right)\right): \tau=(I: \tau)
$$

and

$$
\bigcap_{t}\left(I, \underline{x}^{t}\right)^{*}=I^{*}
$$

(the last equality follows because if $u \in \cap_{t}\left(I, \underline{x}^{t}\right)^{*}$, then $c u^{q} \in \cap_{t}\left(I^{[q]}, \underline{x}^{t q}\right)$ for some $c \in R^{0}$ and all $q=p^{e}$, and so $c u^{q} \in I^{[q]}$ by the Krull intersection theorem, which means that $\left.u \in I^{*}\right)$. It follows that $J(I: \tau) \subset I^{*}$.

Therefore we have $\tilde{I}(I: \tau) \subset I^{*}$, and since the ideal on the right is tightly closed, it follows that $(\tilde{I})^{*}(I: \tau) \subset I^{*}$.

As an application of this result, we prove an unexpected restriction on the linkage class of certain ideals:

Corollary 2 Let $R$ be as above and assume that $I$ is an unmixed ideal containing the test ideal $\tau$. Then $I^{*}=(\tilde{I})^{*}$.

If in addition I is tightly closed, then I contains every ideal in its linkage class. In particular if the test ideal $\tau$ is unmixed and tightly closed, then it is maximal in its linkage class.

If $I, J$ are unmixed ideals containing $\tau$ and are in the same linkage class, then $I^{*}=J^{*}$.

Proof If $I \supset \tau$ then $I: \tau=R$, and Thm. 2 implies that $(\tilde{I})^{*} \subset I^{*}$. If in addition $I$ is tightly closed it follows that $\tilde{I} \subset(\tilde{I})^{*}=I$, and therefore $I$ is maximal in its linkage class. The last statement is immediate, since we have $I^{*}=J^{*}=(\tilde{I})^{*}$.

The next corollary deals with a property of the test ideal:

Corollary 3 Let $(R, \mathbf{m})$ be a Gorenstein ring of characteristic $p$ with test ideal $\tau$; assume that the ideals $\tau$ and $\tau^{2}$ are unmixed. Then we have

$$
\tilde{\tau} \tau=\tau^{2},
$$

where $\tilde{\tau}$ is the sum of all ideals in the linkage class of $\tau$.

Proof Let $\mathbf{a}$ be an ideal generated by parameters, with $\mathbf{a} \subset \tau^{2}$ and of the same height as $\tau$. Apply the result of Thm. 2 for $I=\mathbf{a}: \tau=\mathbf{a}^{*}$. Note that $\tilde{I}=\tilde{\tau}$, and $I$ is tightly closed. We have

$$
\tilde{\tau}\left(\mathbf{a}: \tau^{2}\right) \subset \mathbf{a}: \tau,
$$

or equivalently

$$
\mathbf{a}: \tau^{2} \subset \mathbf{a}: \tilde{\tau} \tau,
$$

and therefore $\tilde{\tau} \tau \subset \tau^{2}+\mathbf{a}=\tau^{2}$ by the choice of $\mathbf{a}$. 
The final application deals with a particular case of the localization problem for tight closure:

Corollary 4 Let $R$ be a Gorenstein ring and let $I$ be an unmixed ideal such $\tilde{I}$ is $\mathbf{m}$-primary. Then for any multiplicative system $W$, we have $\left(I_{W}\right)^{*}=\left(I^{*}\right)_{W}$.

Proof Since $\tilde{I}$ is $\mathbf{m}$-primary, Thm. 2 shows that $(I: \tau) / I^{*}$ is a finite length module and the conclusion follows by Cor. 1 .

Note 4 The hypothesis that $\tilde{I}$ is $\mathbf{m}$-primary is equivalent to $I_{P}$ being in the linkage class of a Gorenstein ideal of finite projective dimension for every prime ideal $P \neq \mathbf{m}$ (choose $J_{n}=\mathbf{a}_{n}:\left(\mathbf{a}_{n-1}: \ldots:\left(\mathbf{a}_{1}: I\right)\right)$ with $n$ minimal such that $J_{n} \not \subset P$. Then $\left(J_{n-1}\right)_{P}=\left(\mathbf{a}_{n-1}\right)_{P}$, which is in the linkage class of $I_{P}$, is a Gorenstein ideal of finite projective dimension).

With the possible exception of the case when $R$ is an isolated singularity (in which case the localization of tight closure holds trivially, because the test ideal is $\mathbf{m}$-primary), it is never the case that the condition discussed above holds for every ideal in $R$, because it implies that $I_{P}$ has finite projective dimension. Moreover, Thm. 2.10 in PU] gives a large class of non $\mathbf{m}$-primary ideals that contain every ideal in their linkage classes, therefore providing examples when the hypothesis of Cor. 1 fails. Note however that the notion considered in $[\mathrm{PU}]$ is linkage by complete intersections; the linkage class by Gorenstein ideals may be larger. Thus, the assumption in Cor. A is quite restrictive, but this author believe it is worth adding to the list of cases when tight closure commutes with localization.

For a concrete non-trivial example when the hypothesis holds, consider a codimension two ideal in a regular local ring, such that $R / I$ is not Cohen-Macaulay, but it becomes Cohen-Macaulay when localized at any non-maximal prime.

\section{References}

Ab. I. Aberbach, Extensions of weakly and strongly F-regular maps, preprint.

Ap. R. Apéry, Sur les courbes de première espèce de l'espace de trois dimensions, C. R. Acad. Sci. Paris, 220 (1945), 271-272.

AN. M. Artin and M. Nagata, Residual intersections in Cohen-Macaulay rings, J. Math. Kyoto Univ. 12 (1972), 307-323.

Ga. F. Gaeta, Détermination de la chaine syzygétique des idéaux matriciels parfaits et son application à la postulation de leurs variétés algêbriques associées, C. R. Acad. Sci. Paris, 234 (1952), 1833-1835.

He. J. Herzog, Ringe der Charakteristik $p$ und Frobeniusfunktoren, Math. Z.,12(1974), 217-248 
HH1. M. Hochster and C. Huneke, Tight closure, invariant theory, and the Briançon-Skoda theorem, J. Amer. Math. Soc., 31990), no. 1, 31-116.

HH2. M. Hochster and C. Huneke, Localization and Test Exponents for Tight Closure, Michigan Math. J., 48 (2000), 305-329.

Hu1. C. Huneke, Tight closure, parameter ideals, and geometry. Six lectures on commutative algebra (Bellaterra, 1996), 187-239, Progr. Math., 166, Birkhuser, Basel, 1998.

Hu2. C. Huneke, Tight closure and its applications, CBMS Regional Conference Series in Mathematics, 88 (1996).

HU. C. Huneke and B. Ulrich, The structure of linkage, Ann. Math. 126 (1987), 277-334.

Mo. P. Monsky, The Hilbert-Kunz function, Math. Ann., 263 (1983), no.1, 43-49.

PU. C. Polini and B. Ulrich, Linkage and reduction numbers, Math. Ann., 310 (1998), 631-651.

PS1. C. Peskine and L. Szpiro, Dimension projective finie et cohomologie locale, Publ. Math. I.H.E.S. 42 (1972), 47-119.

PS2. C. Peskine and L. Szpiro, Liaison des variétés algébriques, Inv. Math. 26 (1974), 271-302.

R. A. P. Rao, Liaison among curves in $\mathbf{P}^{3}$, Inventiones math., 50(1979), 205-217.

Sm. K. E. Smith, Tight closure of parameter ideals, Invent. Math, 115 (1994), 41-60.

Vr. $\quad$ A. Vraciu, Strong test ideals, J. Pure Applied Algebra, 167 (2002), 361373. 